\title{
Análise comparativa da efetividade de métodos avaliativos de cursos técnicos profissionalizantes a distância em Pernambuco
}

\author{
Francisco Kelsen de Oliveira ${ }^{1}$, Leandro Marques Queiros ${ }^{2}$, Alex Sandro Gomes ${ }^{3}$ \\ ${ }^{1}$ Instituto Federal de Educação Ciência e Tecnologia do Sertão Pernambucano (IF \\ Sertão-PE) - Salgueiro, PE - Brasil \\ ${ }^{2}$ Diretoria de Educação a Distância (DEaD) - Instituto Federal de Educação Ciência e \\ Tecnologia de Pernambuco (IFPE) - Recife, PE - Brasil \\ ${ }^{1,2,3}$ Centro de Informática (CIN) - Universidade Federal de Pernambuco (UFPE) - \\ Recife, PE - Brasil \\ francisco.oliveiralifsertao-pe.edu.br, lmq@cin.ufpe.br, asgecin.ufpe.br
}

\begin{abstract}
This research carried out a comparative study between evaluative models adopted in online courses offered by the federal public institutions of education of Pernambuco. The comparative analysis of the methods considered the institutional structure, the model of monitoring of students and the evaluation institutional model. Federal Institutes of Education, Science and Technology of Pernambuco (IFPE) and of Sertão of Pernambuco (IF Sertão-PE) formed the sample of this research. The analysis showed that both evaluation criteria defined were used by teachers of disciplines, while the biggest difference is in the evaluative criteria by IF Sertão-PE institutionalized.
\end{abstract}

Resumo. Esta pesquisa realizou um estudo comparativo entre os modelos avaliativos adotados nos cursos técnicos online ofertados pelas instituições públicas federais de ensino de Pernambuco. Os critérios da análise comparativa dos métodos consideraram a estrutura institucional, o modelo de acompanhamento dos discentes e o modelo avaliativo institucional. Os Institutos Federais de Educação, Ciência e Tecnologia de Pernambuco (IFPE) $e$ do Sertão Pernambucano (IF Sertão-PE) formaram a amostra desta investigação. As análises mostraram que ambas utilizaram critérios avaliativos definidos pelos professores das disciplinas, enquanto a diferença maior está nos critérios avaliativos institucionalizados pelo IF Sertão-PE.

\section{Introdução}

A Educação a Distância (EaD) é definida por Brasil (2005) como modalidade educacional que visa a integração de atividade educativas em lugares ou tempos diversos com o suporte de meios e tecnologias de informação e comunicação. $O$ ensino 
V Congresso Brasileiro de Informática na Educação (CBIE 2016)

Anais dos Workshops do V Congresso Brasileiro de Informática na Educação (CBIE 2016)

a distância utiliza dessas ferramentas para auxiliar o aprendizado do estudante, apropriando-se de recursos eletrônicos, impressos e outros [Moore 1973].

Turrioni e Stano (2009) destacam duas características especiais da EaD em relação a modalidade presencial: uso de tecnologias de informação como fonte principal no processo de aprendizagem e a dificuldade de contato entre professor e aluno. Os autores supracitados consideram que, baseados nessas características, é imprescindível que sejam estudados os parâmetros para a avaliação de cursos em $\mathrm{EaD}$, pois, os métodos tradicionais de avaliação presencial aplicados mostram sinais de desgaste.

As diretrizes e bases da educação nacional do Brasil definem a forma que os estudantes de $\mathrm{EaD}$ devem ser avaliados. A avaliação do desempenho do estudante se dá por meio de cumprimento de atividades programadas e a realização de exames presenciais. O segundo caso prevalece sobre qualquer outra forma de avaliação. Essas avaliações devem estar previstas no projeto pedagógico do curso [Brasil 1996].

A ABED (Associação Brasileira de Educação a Distância) (2015) classifica ações diretas e indiretas no âmbito da $\mathrm{EaD}$, destacando-se as diretas neste artigo, pois estas abrangem os cursos que são ofertados totalmente a distância, blended, híbridos ou semipresenciais e disciplinas realizadas a distância. A forma de avaliação em cada categoria é distinta uma da outra, por exemplo: blended, híbridos ou semipresenciais combinam atividades virtuais e presenciais com proporção que variam de $30 \%$ a $70 \%$ de uma forma em relação a outra [Censo 2015].

Os cursos supracitados estão uma crescente ascensão no Brasil, visto que as instituições de ensino públicas e privadas foram motivadas a expandir essa modalidade de ensino por meio da Lei de diretrizes e Bases (LDB), do ano de 1996 [Brasil 1996]. O Estado de Pernambuco, no ano de 2016, possui duas instituições de ensino públicas federais (IEPF) que oferecem 10 cursos técnicos profissionalizantes gratuitos em EaD.

Dado as considerações de Turrioni e Stano (2009) sobre a necessidade de estudos que visem a melhoria do processo de avaliação em $\mathrm{EaD}$, os diversos modelos de avaliação e suas complexidades, faz-se como objetivo desse estudo realizar um estudo comparativo entre os modelos avaliativos adotados nos cursos técnicos online ofertados pelas instituições públicas federais de ensino do Estado de Pernambuco. Para isso, foi realizado um levantamento exploratório das IEPF que ofertassem cursos técnicos profissionalizantes a distância a fim de analisar os critérios avaliativos de cada instituição.

Esta pesquisa, portanto, apresenta as seguintes seções: aspectos conceituais acerca da EAD, ferramentas de comunicação utilizadas em tal modalidade, metodologia, discussão dos resultados e considerações finais.

\section{Fundamentação Teórica}

A avaliação é indispensável em qualquer modalidade de ensino, pois permite acompanhar se os objetivos de aprendizagem estão sendo atingidos a partir de um conjunto de técnicas, métodos e materiais utilizados.

Bloom, Hastings e Madaus (1983) definem a avaliação como um método de coleta e de processamento dos dados necessários à melhoria da aprendizagem e do 
V Congresso Brasileiro de Informática na Educação (CBIE 2016)

Anais dos Workshops do V Congresso Brasileiro de Informática na Educação (CBIE 2016)

ensino ou ainda como um sistema de controle de qualidade pelo qual se pode determinar, a cada passo do processo ensino-aprendizagem, se este está sendo eficaz ou não.

Nesse sentido, a avaliação pode ser classificada como diagnóstica, somativa e formativa [Abreu 2011]. A avaliação diagnóstica é a sondagem inicial estabelecida entre o professor e o aprendiz a fim de detectar os conhecimentos desse para escolha de recursos, técnicas e métodos didáticos a serem mais bem utilizados no processo de aprendizagem [Bordón 2004; Eres Férnandez e Baptista 2010]. Enquanto a avaliação somativa ou acumulativa averigua os conhecimentos ou nível do aprendiz em relação aos objetivos estabelecidos [Hoffmann 2005; Bordón 2004]. Já a formativa ou processual busca analisar o processo de formação e de reflexão do trabalho feito e aprendido de forma conjunta entre professores e alunos [Eres Férnandez e Baptista 2010].

No âmbito da EaD, conforme Martins et al. (2010), a avaliação formativa é possível de ser realizada a partir das diversas ferramentas síncronas e assíncronas existentes no Ambiente Virtual de Aprendizagem (AVA) no decorrer do curso ou disciplina ao invés de avaliar o aluno em um único momento, muitas vezes, apenas no final da disciplina, quando não há tempo suficiente para identificar as deficiências de aprendizagem dos alunos e buscar as tentativas de solucioná-las. Contudo, seja qual for a modalidade de ensino, as avaliações a partir de ferramentas dos AVAs devem ter critérios avaliativos claros e inerentes às características da referida ferramenta, para que coadune o objetivo de aprendizagem estabelecido com a referida função que levou a sua inclusão naquele contexto educacional.

Os cursos e disciplinas são planejados com base em uma proposta pedagógica que favoreça a construção de estratégias motivadoras da participação dos alunos e forneçam os subsídios da avaliação [Fiorentini 2002]. Segundo Martins et al. (2010), essas propostas metodológicas podem envolver os seguintes espaços pedagógicos: AVAs, encontro presencial (EP), material didático (MD), acompanhamento e apoio (AA) e critérios avaliativos de aprendizagem (CAA).

Deve-se contemplar em um contexto atual não apenas AVAs, mas os sistemas com funções necessárias para o gerenciamento e oferta de cursos e conteúdos educacionais, como Redes Sociais Educacionais (RSE). De modo geral, espera-se que essas aplicações contemplem ferramentas síncronas (chat, webconferência e videoconferência) e assíncronas (fóruns, mensagens, portfólios e questionários), nas quais possam gerenciar recursos didáticos, usuários e suas interações.

Os EPs possibilitam o fortalecimento dos vínculos entre docentes, discentes e tutores, garantem a realização de atividades práticas, principalmente, em cursos técnicos e cumprem as determinações legais do decreto $\mathrm{n}^{0} 5.622$ e resolução $\mathrm{n}^{0} 6$, de 2012 , do Ministério da Educação (MEC), que apresentam a necessidade da existência mínima de $20 \%$ de atividades presenciais nos cursos a distância [Brasil 2005].

Os MDs proporcionam leituras aos alunos acerca dos conteúdos das disciplinas e geralmente são disponibilizados arquivos digitais em formato PDF ou mesmo são impressos e distribuídos aos alunos ou disponibilizados nas bibliotecas dos polos dos cursos. A elaboração desses materiais oportuniza os estudos dos discentes em qualquer 
lugar mesmo sem conexão à Internet. Enfatiza-se também que muitas instituições mantêm equipes de profissionais capacitados para produzirem materiais ricos em conteúdos, com uma linguagem clara e objetiva, bem como com um layout agradável para proporcionar uma boa experiência de leitura. No caso de cursos da Rede e-Tec Brasil, há uma biblioteca com livros das disciplinas em formato PDF para que as instituições possam utilizar em cursos ofertados em parceria com a Rede e-Tec.

O acompanhamento e apoio (AA) são realizados pela equipe de profissionais responsáveis pelos cursos, que envolve desde tutores a distância (TA), tutores presenciais (TP) e professores das disciplinas. Há modelos diferentes de atuação desses profissionais, de acordo com cada instituição. No entanto, a atuação deles em conjunto com o envio de mensagens informativas, sanando dúvidas dos discentes, planejando aulas e materiais colaboram para a oferta e realização dos cursos.

Os critérios avaliativos de aprendizagem (CAA) são os aspectos considerados para cada atividade realizada no curso a fim de deixar claro quais são os pontos levados em consideração no alcance dos objetivos didáticos. Os CAA também sofrem alterações de acordo com instituições, cursos e até mesmo em disciplinas diferentes de um mesmo curso, porque devem atender as especificidades avaliativas de cada curso.

\section{Método}

Esta pesquisa realiza uma análise comparativa de métodos avaliativos utilizados em cursos técnicos profissionalizantes ofertados na modalidade a distância das IEPF do Estado de Pernambuco, assim a amostra foi composta por duas instituições: Institutos Federais de Educação, Ciência e Tecnologia de Pernambuco (IFPE) e do Sertão Pernambucano (IF Sertão-PE).

Ambas instituições ofertam seus cursos técnicos a distância em parcerias com o programa e-Tec, seguem padrões semelhantes nos formatos de atuações dos profissionais e estão em conformidade com as diretrizes do catálogo nacional de cursos técnicos [Brasil 2016].

O IFPE oferece os seguintes cursos técnicos em seis municípios pernambucanos: Manutenção e Suporte em Informática (MSI), Alimentação Escolar (AE), Informática para Internet (IPI), Infraestrutura Escolar (IE), Manutenção Automotiva (MAV) e Sistemas de Energia Renovável (SER). Esses cursos são regidos pela Organização Acadêmica Institucional (OAI), a qual delineia as normas, procedimentos, orientações e diretrizes pertinentes a vida acadêmica institucional dos discentes e docentes [Brasil 2014a].

O IF Sertão-PE oferta os cursos técnicos de MSI, Serviços Públicos, Segurança do Trabalho (ST), Logística e Agente Comunitário de Saúde (ACS) distribuídos em sete polos. Esses cursos seguem a Organização Didática Geral da instituição que regulamenta aspectos de frequências, notas e médias de aprovação e reprovação [Brasil 2010]. A Coordenação de EAD (CEAD) elaborou dois documentos: Referencial Metodológico de EAD [Brasil 2013] e os critérios avaliativos. Esses critérios foram criados especificamente para os quatro últimos cursos da instituição, por isso que esta pesquisa levará em consideração apenas a esses cursos do IF Sertão-PE, já que MSI adota modelo similar ao mesmo curso em IFPE. 
V Congresso Brasileiro de Informática na Educação (CBIE 2016)

Anais dos Workshops do V Congresso Brasileiro de Informática na Educação (CBIE 2016)

Conforme a classificação de Appolinário (2016), esta investigação pode ser classificada conforme os seguintes aspectos: finalidade (aplicada), tipo/profundidade (descritiva), estratégia para origem dos dados (a partir dos documentos institucionais), natureza (qualitativa) e temporalidade (transversal). Os aspectos considerados nas análises estão baseados em adaptações das considerações de Martins et al. (2010) sobre os espaços pedagógicos, Quadro 1.

Quadro 1 - Aspectos a serem considerados nas análises dos documentos institucionais.

\begin{tabular}{|c|c|c|c|}
\hline ID & Aspectos Gerais & $\begin{array}{l}\text { Aspectos } \\
\text { Específicos }\end{array}$ & Descrição \\
\hline A1 & $\begin{array}{l}\text { Ambiente Virtual } \\
\text { de Aprendizagem } \\
(\text { AVA) }\end{array}$ & & $\begin{array}{l}\text { Possui AVA, RSE ou outro sistema de gerenciamento de } \\
\text { aprendizagem previamente configurado e disponível para oferta de } \\
\text { cursos. }\end{array}$ \\
\hline A2 & $\begin{array}{l}\text { Encontro } \\
\text { Presencial (EP) }\end{array}$ & & $\begin{array}{l}\text { Os EPs são realizados nos polos presenciais com o suporte do tutor } \\
\text { presencial (TP), pessoa responsável de prestar suporte aos discentes e } \\
\text { quantidade de EPs de acordo com a carga-horária de cada } \\
\text { componente curricular, sendo no mínimo um EP. }\end{array}$ \\
\hline A3a & $\begin{array}{l}\text { Material didático } \\
(\mathrm{MD})\end{array}$ & Origem & $\begin{array}{l}\text { Oferta MDs específico aos cursos existentes na instituição produzidos } \\
\text { da instituição ou utiliza materiais de terceiros. }\end{array}$ \\
\hline $\mathbf{A} 3 \mathbf{b}$ & & Formato & MDs estão disponíveis em formatos digitais e físicos. \\
\hline A3c & & Tipos & MDs são elaborados em pelo menos dois: texto, vídeo e áudio. \\
\hline A3d & & Produção & $\begin{array}{l}\text { MDs são produzidos por professor ou por equipe de produção } \\
\text { formada por designers instrucionais, pedagogos e docentes. }\end{array}$ \\
\hline A3e & & Avaliação & $\begin{array}{l}\text { MDs elaborados são analisados por equipe multidisciplinar, por pares } \\
\text { ou pelo coordenador de curso. }\end{array}$ \\
\hline A4 & $\begin{array}{l}\text { Acompanhamento } \\
\text { e apoio (AA) }\end{array}$ & & $\begin{array}{l}\text { Há tutores presenciais (TP) ou a distância (TD) e professores para } \\
\text { acompanhamento dos alunos. Esses profissionais possuem atribuições } \\
\text { definidas para atuarem nos cursos. }\end{array}$ \\
\hline A5a & $\begin{array}{l}\text { Critérios } \\
\text { avaliativos } \\
\text { aprendizagem } \\
\text { (CAA) }\end{array}$ & Chats & $\begin{array}{l}\text { - Existem critérios avaliativos institucionais ou por disciplina } \\
\text { definidos para tal atividade. } \\
\text { - Professor ou TD sanam as dúvidas dos alunos pelo AVA de forma } \\
\text { síncrona ou discute temas propostos pelo mediador. }\end{array}$ \\
\hline A5b & & Fórum & $\begin{array}{l}\text { - Existem critérios avaliativos institucionais ou por disciplina } \\
\text { definidos para tal atividade. } \\
\text { - Discussão sobre tema proposto a partir do assunto em estudo e } \\
\text { mediada por professor, TP ou TD. }\end{array}$ \\
\hline A5c & & $\begin{array}{l}\text { Atividade } \\
\text { Presencial } \\
\text { (AP) }\end{array}$ & $\begin{array}{l}\text { - Existem critérios avaliativos institucionais ou por disciplina } \\
\text { definidos para tal atividade. } \\
\text { - Atividade individual ou em grupo realizada no polo de apoio } \\
\text { presencial e entregue pelo AVA ou fisicamente. }\end{array}$ \\
\hline A5d & & $\begin{array}{l}\text { Atividade } \\
\text { Virtual } \\
\text { (AV) }\end{array}$ & $\begin{array}{l}\text { - Existem critérios avaliativos institucionais ou por disciplina } \\
\text { definidos para tal atividade. } \\
\text { - Atividade individual, em grupo ou colaborativa de autoria(s) do(s) } \\
\text { aluno(s) realizada exclusivamente pelo AVA, que pode ser: envio de } \\
\text { arquivo, fórum, questionário, wiki e outros. }\end{array}$ \\
\hline A5e & & $\begin{array}{l}\text { Avaliação } \\
\text { presencial } \\
\text { individual } \\
\text { (API) }\end{array}$ & $\begin{array}{l}\text { - Existem critérios avaliativos institucionais ou por disciplina } \\
\text { definidos para tal atividade. } \\
\text { - Avaliação realizada no polo de apoio presencial de caráter } \\
\text { obrigatório com variação conforme cada disciplina. }\end{array}$ \\
\hline
\end{tabular}

Buscou-se, então, perceber as possíveis relações existentes entre os instrumentos avaliativos utilizados em cada um e as métricas de avaliação utilizadas. Após a identificação das IEPFs, foi realizada busca por documentos institucionais nas páginas eletrônicas a fim de encontrar regimentos, normas e regulamentos que tratassem e explicitassem os métodos avaliativos institucionais utilizados nos cursos técnicos a distância, que respondessem aos aspectos descritos no Quadro 1, cujos resultados serão apresentados a seguir. 
V Congresso Brasileiro de Informática na Educação (CBIE 2016)

Anais dos Workshops do V Congresso Brasileiro de Informática na Educação (CBIE 2016)

\section{Resultados e discussões}

A instituição IFPE adota o processo de avaliação estabelecido da sua própria OAI. O processo de avaliação de aprendizagem busca acompanhar o estudante de forma contínua e integral. Esse acompanhamento se dá por meio das atividades programadas a distância, as quais acontecem ao longo das semanas em que o componente curricular (CC) está distribuído. Dois ou mais CCs podem ser executados simultaneamente, ou seja, não modular. As atividades virtuais via AVA e a participação no AVA, o qual tem como base o CAA, são desenvolvidas semanalmente de forma ininterrupta. As avaliações presenciais são criadas pelo professor formador, e desenvolvidas nos polos presenciais.

O estudante pode realizar a AP se, e somente se, tiver participado de, no mínimo, uma Atividade Programada a Distância (APD), obtendo nota diferente de 0,0 (zero). Há previsão da Avaliação de Segunda Chamada (SC) para o aluno que tiver se ausentado de atividade acadêmica. Essa será concedida após o estudante apresentar justificativa, conforme previsões da OAI. Nesse sentido, a composição da avaliação de aprendizagem e seus respectivos pesos podem ser visualizados no Quadro 2.

Quadro 2 - Processo de Avaliação da Aprendizagem da EaD na Instituição IFPE.

\begin{tabular}{lcl}
\hline Avaliações & Peso (\%) & Composição da Avaliação \\
\hline Atividades Programadas a Distância (APD) & 30 & $\begin{array}{l}\text { - Atividades Virtuais (20\%) } \\
\text { - Participação no AVA (10\%) }\end{array}$ \\
\hline Avaliações Presenciais (AP) & $70 \quad \begin{array}{l}\text { - Encontro Presencial }(20 \%) \\
\text { - Avaliação Presencial ou SC (50\%) }\end{array}$ \\
\hline Média das Avaliações Realizadas (MAR) & - MAR = APD + AP (100\%) \\
\hline & Fonte: [Brasil 2014a].
\end{tabular}

O aluno está aprovado se MAR for maior ou igual 6,0. Aquele que obtiver nota entre 5,9 e 2,0 poderá realizar o Exame Final, contudo, MAR menor que 2,0 está automaticamente reprovado, conforme a composição da Média Final no Quadro 3.

Quadro 3 - Composição da Média Final da EaD na Instituição IFPE.

\begin{tabular}{lll}
\hline Média & Cálculo & Observação \\
\hline Exame Final (EF) & $\begin{array}{l}\text { Nota obtida após } \\
\text { realização do EF }\end{array}$ & $\begin{array}{l}\text { O aluno que obtiver nota menor que 2 ou maior que 6 não poder } \\
\text { realizar o EF. }\end{array}$ \\
\hline Média Final (MF) & MF $=(\mathrm{MAR}+\mathrm{EF}) / 2$ & Aluno era aprovado mediante MF nota igual ou superior a 6,0. \\
\hline & & Fonte: [Brasil 2014a].
\end{tabular}

O IF Sertão-PE segue um padrão semelhante ao que Martins et al. (2010), o qual é bem parecido ao modelo avaliativo de IFPE, pois trabalha com atividades presenciais e a distância baseadas nas funcionalidades no AVA utilizado pela instituição, Modular Object-Oriented Dynamic Learning Environment (Moodle). No entanto, cada atividade possui nota e frequência atribuída, que ao final das disciplinas comporão as notas a serem incluídas no sistema de controle acadêmico institucional (SCAI), ver Quadro 4.

Cada disciplina ocorre de forma modular, assim durante o período de vigência da disciplina o aluno se dedica integralmente a um conteúdo com atividades virtuais e presenciais definidas pelo professor da disciplina e em consonância com o modelo de processo avaliativo da aprendizagem adotado pela instituição, conforme Quadros 4 e 5. 
V Congresso Brasileiro de Informática na Educação (CBIE 2016)

Anais dos Workshops do V Congresso Brasileiro de Informática na Educação (CBIE 2016)

Quadro 4 - Processo de Avaliação da Aprendizagem da EaD na Instituição IF Sertão-PE.

\begin{tabular}{|c|c|c|c|}
\hline Avaliação & Peso (\%) & Composição da Avaliação & Frequência (hora/aula) \\
\hline \multirow{4}{*}{$\begin{array}{l}\text { Notas de Atividades } \\
\text { Programadas a } \\
\text { distância (NAPD) }\end{array}$} & \multirow[t]{4}{*}{30} & Fórum $1(25 \%)$ & 6 \\
\hline & & Fórum $2(25 \%)$ & 10 \\
\hline & & Chat $(25 \%)$ & 6 \\
\hline & & Portfólio $(25 \%)$ & 10 \\
\hline \multirow{2}{*}{$\begin{array}{l}\text { Notas de Exames } \\
\text { Presenciais (NEP) }\end{array}$} & \multirow[t]{2}{*}{70} & Avaliação presencial em grupo (APG) [20\%] & 4 \\
\hline & & Avaliação presencial individual (API) [80\%] & 4 \\
\hline Nota Final (NF) & $*$ & Avaliação presencial individual final (APIF) & 4 \\
\hline
\end{tabular}

Além do estabelecimento das atividades técnicas a serem realizadas pelas ferramentas do AVA, havia critérios avaliativos específicos para nortear docentes e discentes em IF Sertão-PE com a definição de pontuação por critérios, ver Quadro 5.

Quadro 5 - Critérios avaliativos por atividades da EaD na Instituição IF Sertão-PE.

\begin{tabular}{|c|c|c|c|}
\hline $\begin{array}{l}\text { Atividade } \\
\text { s }\end{array}$ & Critérios & Descrições & Pontos \\
\hline \multirow[t]{3}{*}{ Fórum 1} & Participação & $\begin{array}{l}\text { Apresentar a experiência na área, o motivo da escolha do curso, as } \\
\text { expectativas e proposição dos horários de estudo para disciplina. }\end{array}$ & 6 \\
\hline & \multirow[t]{2}{*}{ Textual } & Coesão & 2 \\
\hline & & Coerência & 2 \\
\hline \multirow[t]{5}{*}{ Fórum 2} & \multirow[t]{3}{*}{ Participação } & $\begin{array}{l}\text { Participação frequente com comentários e questões relevantes que } \\
\text { contribuam para o tópico da discussão. }\end{array}$ & 2 \\
\hline & & $\begin{array}{l}\text { Intervenção nas mensagens dos outros participantes com comentários e } \\
\text { questionamentos que instiguem o debate. }\end{array}$ & 2 \\
\hline & & $\begin{array}{l}\text { Inserção de comentários próprios, ao invés de apenas repetir as } \\
\text { colocações de outros autores. }\end{array}$ & 2 \\
\hline & \multirow[t]{2}{*}{ Textual } & Coesão & 2 \\
\hline & & Coerência & 2 \\
\hline \multirow[t]{2}{*}{ Chat } & Participação & Participou e interagiu com outro(s) aluno(s) sobre o tema proposto. & 10 \\
\hline & & $\begin{array}{l}\text { Não participou ou participou com mensagens vazias de sentido ou } \\
\text { desassociadas ao tema discutido, que não contribuíram para o processo de } \\
\text { comunicação e aprendizagem. }\end{array}$ & 0 \\
\hline
\end{tabular}

Enfatiza-se que os critérios de "participação" apresentados no Quadro 5 buscaram estimular a participação dos alunos durante as disciplinas, assim como incentivar as participações discentes entre eles e entre os discentes e docentes, de tal forma a promover o diálogo com base nos conteúdos, conforme Freire, David e Oliveira (2011). Outro ponto de destaque ainda no critério participação no Fórum 1 está na proposição de um plano de estudo para a disciplina, que possibilita o planejamento do professor para marcar chats, preferencialmente, nos horários definidos pela maioria dos alunos, bem como garantir uma espécie de contrato didático entre docente e discente para acompanhar a disciplina em horários de estudo previamente estabelecidos. Outra ênfase importante nos critérios de IF Sertão-PE está no aspecto textual como forma de promover a escrita de acordo com a norma culta da língua portuguesa e evitar o uso de gírias ou de linguagem informal usada em salas de bate-papo na Internet.

As disciplinas desses cursos do IF Sertão-PE tinham 40 h/a, assim a realização de cada atividade garantia também a frequência na disciplina. Esse foi um fator em destaque do IFPE em relação a IF Sertão-PE, pois as realizações das atividades baseadas em critérios avaliativos previamente estabelecidos também garantiram a frequência dos alunos na disciplina. Em relação à composição das notas, IFPE e IF Sertão-PE trabalham de formas análogas, porém os discentes do IF Sertão-PE poderiam 
V Congresso Brasileiro de Informática na Educação (CBIE 2016)

Anais dos Workshops do V Congresso Brasileiro de Informática na Educação (CBIE 2016)

ter uma avaliação de recuperação (APIF) de nota e frequência apenas da API para quem estiver de acordo com a condição 2 do Quadro 6.

Quadro 6 - Composição das médias da EaD na Instituição IF Sertão-PE.

\begin{tabular}{lll}
\hline Média & Cálculo & Observação \\
\hline $\begin{array}{l}\text { Média } \\
\text { Global (MG) }\end{array}$ & NAPD + & $\begin{array}{l}\text { Condição 1: Aluno aprovado com MG maior ou igual a 6,0 (seis) e frequência maior } \\
\text { ou igual a 75\%. Condição 2: Aluno com MG entre 5,9 e 3,0 e frequência maior ou } \\
\text { igual a 75\% pode realizar APIF. Condição 3: Aluno reprovado com MG menor que } \\
\text { 3,0 ou frequência inferior a 75\%. }\end{array}$ \\
\hline $\begin{array}{l}\text { Média Final } \\
\text { (MF) }\end{array}$ & $\begin{array}{l}\text { Condição 4: Aluno aprovado mediante MPF igual ou superior a } 6,0 \text { (seis) e } \\
\text { frequência na disciplina igual ou superior a 75\%. }\end{array}$ \\
\hline
\end{tabular}

Fonte: [Brasil 2010].

A identificação dos aspectos de ID A1 à A5e do Quadro 1 possibilitou a elaboração do Quadro 7 com as seguintes classificações: "Sim", que corresponde ao atendimento integral do critério pelas instituições em estudo, "Não", quando não foi identificado ou "Parcialmente" para o não atendimento e representado pela letra "P".

Quadro 7 - Análise dos aspectos considerados para avaliação em EaD.

\begin{tabular}{lllllllllllllcc}
\hline ID & A1 & A2 & A3a & A3b & A3c & A3d & A3e & A4 & A5a & A5b & A5c & A5d & A5e \\
\hline IFPE & Sim & Sim & Sim & P & P & P & P & Sim & P & P & P & P & P \\
\hline IF Sertão-PE & Sim & Sim & Sim & P & P & P & P & Sim & Sim & Sim & Sim & Sim & Sim \\
\hline \multicolumn{1}{c}{ Fonte: Pesquisa direta. }
\end{tabular}

As instituições possuem instâncias do AVA Moodle disponíveis, um plano de EP agendados para cada disciplina e estruturas de AA semelhantes. Dessa forma, atendem, respectivamente, aos critérios $\mathrm{A} 1, \mathrm{~A} 2$ e A4. Já em relação aos critérios $\mathrm{A} 3 \mathrm{a}$ à A3e, ambas instituições atendem parcialmente. Em A3a, por exemplo, não há padronização na produção dos materiais, assim alguns são disponibilizados a partir da biblioteca do e-Tec, enquanto outros são elaborados pelos professores das disciplinas. Já em A3b, IF Sertão-PE garante apenas MD em formatos digitais, principalmente, em PDF para todas as disciplinas, enquanto IFPE possui disciplinas que não são apresentados MD.

Dessa forma, percebe-se que o critério A3c também é atendido parcialmente em ambas, porque nem todas as disciplinas de ambas possuem vídeos sobre os assuntos, enquanto os MDs em PDF não estão disponíveis obrigatoriamente em todas as disciplinas de IFPE. Os critérios A3d e A3e também são parcialmente atendidos por ambas, porque os MD produzidos nas respectivas instituições são elaborados pelos professores sem acompanhamento de uma equipe multidisciplinar para dar suporte às produções do docente, enquanto as avaliações dos materiais produzidos são geralmente realizadas apenas pelos coordenadores dos cursos.

Já os critérios A5a e A5e são cumpridos integralmente em IF Sertão-PE, por causa das descrições dos critérios avaliativos de cada tipo de atividade a serem utilizados nas disciplinas juntamente outros critérios específicos das disciplinas e apresentados pelos professores autores dos MDs, enquanto IFPE basicamente trabalha apenas com aspectos planejados pelos professores das disciplinas.

O estabelecimento de critérios avaliativos institucionais possibilita atingir um padrão de qualidade em que toda equipe esteja ciente e engajada no que se almeja em cada disciplina. Além disso, os discentes ficam cientes de como serão avaliados e quais aspectos podem melhorar no decorrer da disciplina e no curso. No entanto, não basta 
apenas estabelecer os critérios, pois as atividades de apoio e acompanhamento desses critérios e das ações dos docentes no cumprimento desses critérios e dos discentes na verificação de suas dificuldades e alcance dos objetivos de aprendizagem são essenciais para se chegar a um alto padrão de qualidade dos cursos.

Portanto, os modelos avaliativos utilizados pelas instituições pesquisadas estão baseados nos aspectos formativos que possibilitam o acompanhamento discente no decorrer da disciplina, assim é possível que o planejamento realizado pelo docente possa se adequar às necessidades específicas de um determinado grupo ou polo, porém, requer o acompanhamento sistemático da equipe envolvida com tais cursos.

\section{Considerações finais}

Observou-se que os critérios estabelecidos nesta pesquisa são apresentados em ambas instituições, porém, deve-se analisar cuidadosamente a possibilidade de adequação de MDs às necessidades das instituições, cursos, docentes e discentes. Isso pode ser feito até mesmo com adequações dos materiais propostos pelo programa e-Tec.

A OAI de IFPE prevê nota ao aluno por frequência nos cursos da $\mathrm{EaD}$, na qual essa tem que ser igual ou superior $75 \%$ no computo da carga horária total dos CCs para que o aluno seja aprovado, porém, não foi identificado campo onde esta nota fosse efetivamente computada. Logo, o aluno não precisa comparecer ao(s) EP(s) de cada CC para que possa obter a aprovação no CC. Tal fato pode ser um dos fatores influenciadores na evasão dos estudantes, haja vista que o Centro Estadual de Educação Tecnológica Paula Souza (2013) considera que os cursos técnicos têm níveis altíssimos de evasão, especialmente os de informática, que giram em torno de $40 \%$.

Embora critérios avaliativos institucionalizados sejam importantes para garantir a padronização da avaliação e alcance de padrões de qualidades dos cursos, fatores específicos dos cursos devem considerar e coadunar com aspectos institucionais e legais para que efetivamente possam ser adequados às necessidades de formações das áreas dos cursos. Alguns trabalhos futuros podem ser propostos a partir desta investigação: (i) realizar estudo sobre a evasão em cursos técnicos a partir da percepção dos alunos, professores, coordenadores e equipe multidisciplinar; (ii) analisar os resultados dos discentes de cursos técnicos sob diferentes modelos avaliativos; e (iii) verificar e propor modelos avaliativos adequados aos cursos técnicos do programa e-Tec.

\section{Referências}

Abreu, K. F. (2011). Concepções de leitura e de texto subjacentes às provas de vestibular: constatações e implicações para o ensino da língua espanhola. Dissertação - Universidade Federal do Ceará, Programa de Pós-Graduação em Linguística, Fortaleza.

Appolinário, F. (2016). Metodologia da ciência: filosofia e prática da pesquisa. 2. Ed. São Paulo: Cengage Learning.

Bloom, B.; Hastings, T.; Madaus, G. (1983). Manual de avaliação formativa e somativa do aprendizado escolar. São Paulo: Pioneira.

Bordón, T. (2004). Panorama histórico del desarrollo de algunas de las cuestiones fundamentales en la evaluación de segundas lenguas. In: Carabela, 55. Monográfico. 
V Congresso Brasileiro de Informática na Educação (CBIE 2016)

Anais dos Workshops do V Congresso Brasileiro de Informática na Educação (CBIE 2016)

La evaluación en la enseñanza de español como segunda lengua/lengua extranjera. Madrid: Sociedad general española de librería, S.A.

Brasil. (1996). Lei 9394/96 - Lei de Diretrizes e Bases da Educação Nacional.

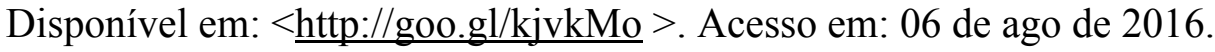

Brasil. (2005). Decreto 5.622 de 19 de dezembro de 2005. Disponível em:

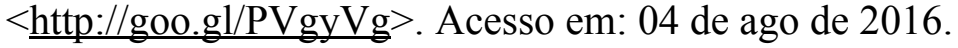

Brasil (2010). Resolução $n^{o} 40$ do Conselho Superior de 21 de dezembro de 2010 -

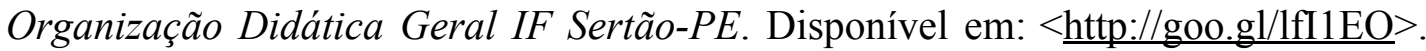
Acesso em: 01 de ago de 2016.

Brasil (2013). Referencial Metodológico de EAD - IF Sertão-PE. Petrolina: IF Sertão-PE.

Brasil. (2014a). Organização Acadêmica Institucional. Disponível em:

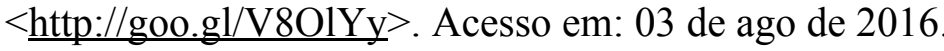

Brasil. (2014b). Orientação acadêmica para cursos técnicos EaD. Disponível em: $<$ http://goo.gl/V8O1Yy $>$. Acesso em: 03 de ago de 2016.

Brasil. (2016). Catálogo Nacional de Cursos Técnicos. Ministério da Educação, $3^{\mathrm{a}}$

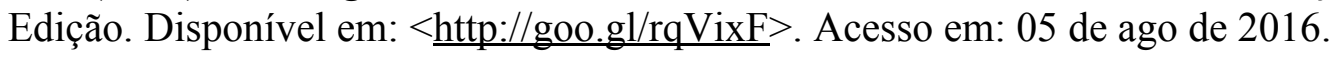

Censo, E. (2015). BR. Relatório analítico da aprendizagem a distância no Brasil 2014, Organização Associação Brasileira de Educação a Distância. Curitiba: Ibpex.

Centro Estadual de Educação Tecnológica Paula Souza. (2013). Plano de Curso Informática. Disponível em: http://goo.gl/4QWBwY. Acesso em: 07 de ago de 2016.

Eres Fernández, I.G.M; Baptista, L.M.T.R. (2010). La enseñanza de lenguas extranjeras y la evaluación. Madrid: Arco/Libros, S.L.

Fiorentini, L. M. R. (2002). Materiais didáticos escritos nos processos formativos a distância. In: Congresso de Ensino Superior a Distância, I, 2002. Petrópolis. Anais. Petrópolis: EsuD.

Freire, R. S.; David, P. B.; Oliveira, F. K. (2011). Dialogicidade na Formação Online de Professores de Matemática. In: XXII Simpósio Brasileiro de Informática na Educação, 2010. Aracaju-SE. Anais do XXII SBIE WIE. Porto Alegre: SBC.

Hoffmann, J. (2005). O jogo do contrário em avaliação. Porto Alegre: Mediação.

Martins, C. A.; Vasconcelos, F. H. L.; Oliveira, F. K.; Santana, J. R.; Sousa, M. I. P. (2010). Dinâmica Pedagógica em Educação a Distância: Caracterizando Aspectos Metodológicos de uma Disciplina de Graduação da UAB. In: Workshop Brasileiro de Informática na Educação. João Pessoa-PB. Anais do Workshop Brasileiro de Informática na Educação. Porto Alegre: SBC.

Moore, M. G. (1973). Toward a theory of independent learning and teaching. In The Journal of Higher Education, pages 661-679.

Turrioni, A. M. S., \& Stano, R. C. M. T. (2009). Critérios de avaliação para a educação a distância. In XXII Congresso Internacional EDUTEC, Manaus. 